\title{
A Gap Theorem For Complete Noncompact Manifolds With Nonnegative Ricci Curvature
}

\author{
Bing-Long Chen AND XI-Ping ZhU ${ }^{1}$
}

In this paper we obtain a gap theorem for the Ricci curvature over complete noncompact locally conformally flat manifolds. We prove that if the Ricci curvature is nonnegative and decays faster than quadratic at infinity, then the manifold is flat.

\section{Introduction.}

Let $M^{n}$ be an n-dimensional complete noncompact Riemannian manifold with nonnegative (sectional or Ricci) curvature. We are interested in the question that how much the curvature could have near the infinities. In views of the classical Bonnet-Meyers theorem there cannot too much positive curvature there otherwise the manifold would close up, ceasing to be noncompact. At first sight, there seems to be no restriction in the other direction, no limit on how less positive curvature can have. For example, it is easy to construct complete metrics on $\mathbf{R}^{2}$ from surface of revolution such that their curvatures are zero outside some compact set, nonnegative everywhere, and positive somewhere. But it is rather surprising that the corresponding situation for a complete manifold $M^{n}$ with dimension $n>2$, can not occur. In [MSY] Mok, Siu and Yau proved that if a complete noncompact Kähler-Stein manifold of nonnegative holomorphic bisectional curvature of complex dimension $m \geq 2$ has maximal volume growth and the scalar curvature decays faster than quadratic, in the sense that, for some $C>0$ and $\varepsilon>0$,

$$
\sup R(x) \leq C r^{-(2+\varepsilon)}
$$

where the supremum of the scalar curvature is taken over all points $\mathrm{x}$ at distance $\mathrm{r}$ from a fixed point, then $\mathbf{M}$ is isometrically biholomorphic to $\mathrm{C}^{m}$.

\footnotetext{
${ }^{1}$ Research was partially supported by Fundation for Outstanding Young Scholars, the NSF of Guangdong Province and China.
} 
This result shows that there is a gap between the flat metric and the other metrics of nonnegative curvature on $\mathbf{C}^{m}$. Subsequently, Greene and $\mathrm{Wu}[\mathrm{GW}]$ studied the corresponding, more general Riemannian situation and proved that a manifold with a pole, with faster than quadratic curvature decay (1.1), and with nonnegative sectional curvature is necessarily flat, if the dimension of the manifold is $\geq 3$ except when the dimension is 4 or 8 . Recently the pole assumption was removed by Eschenburg, Schroeder and Strake [ESS] in the odd-dimensional case and by Dress [Dr] in the evendimensional case.

Naturally one would ask if there are similar gap phenomena for the Ricci curvature, instead of the sectional curvature, over complete noncompact Riemannian manifolds. In general, this seems no longer possible because there are many nonflat Ricci-flat metrics, for examples, the Eguchi-Hanson metric described in $[\mathrm{P}]$ and the Ricci-flat complete Kähler manifolds constructed in [TY]. The Eguchi-Hanson metric has Ricci $\equiv 0,\left|R_{m}\right| \leq \frac{C}{r^{6}}$, and $\operatorname{vol} B(x, r) \geq \frac{1}{2} \omega_{n} r^{n}$ for all $x$, where $\omega_{n}$ is the volume of the unit sphere in the Euclidean space. However, Bando,Kasue and Nakajima [BKN] could still get such a gap result for the Ricci curvature on a complete noncompact Riemannian manifold with maximal volume growth. More precisely, they proved in $[\mathrm{BKN}]$ that:

Suppose a complete noncompact Riemannian manifold $M^{n}$ of dimension $n \geq 3$ satisfies

$$
\left\{\begin{array}{cl}
\text { Ricci } \geq 0, & \\
\operatorname{vol}\left(B\left(x_{0}, r\right)\right) \geq \gamma \omega_{n} r^{n}, & \text { for some } \gamma>\frac{1}{2}, x_{0} \in M^{n}, \text { and all } r>0 \\
\sup |k(\sigma)| \leq C r^{-2}, & \text { if } r \text { is sufficiently large },
\end{array}\right.
$$

and either

$$
(\sup |k(\sigma)|) \cdot r^{-2} \rightarrow 0, \quad \text { as } r \rightarrow+\infty
$$

or

$$
\int_{M^{n}}\left|R_{m}\right|^{\frac{n}{2}} d v<+\infty,
$$

where the supremum of the sectional curvature is taken over all two-planes $\sigma$ at distance $r$ from $x_{0}$ and $R_{m}$ is the curvature operator. Then $M^{n}$ is isometric to Euclidean space $\mathbf{R}^{n}$.

The argument in $[\mathrm{BKN}]$ is via the Gromov convergence theorem. The maximal volume growth assumption which guarantees non-collapsing limit is crucial for their method. Moreover in view of the Eguchi-Hanson metric the assumption that the volume growth factor $\gamma>\frac{1}{2}$ is necessary for the 
above theorem. Thus it is interesting to investigate the gap phenomena on non-maximal volume growth manifolds. In the recent work $[\mathrm{N}], \mathrm{Ni}$ gave some of such gap results on complete noncompact Kähler manifolds. In particular, Mok-Siu-Yau's gap theorem on maximal volume growth manifolds was extended to non-parabolic manifolds in $[\mathrm{N}]$. The purpose of this present paper is also to provide such a gap phenomena for the Ricci curvature over non-maximal volume growth manifolds. The main result which we prove is the following:

Main Theorem. Let $M^{n}$ be an $n$-dimensional $(n \geq 3)$ complete noncompact locally conformally flat manifold with nonnegative Ricci curvature. Suppose the scalar curvature is bounded and there exists a positive function $\varepsilon: \mathbf{R} \rightarrow \mathbf{R}$ with $\lim _{r \rightarrow \infty} \varepsilon(r)=0$ such that

$$
\frac{1}{\operatorname{vol}\left(B\left(x_{0}, r\right)\right)} \int_{B\left(x_{0}, r\right)} R(x) d v \leq \frac{\varepsilon(r)}{r^{2}}, \quad \text { for } x_{0} \in M^{n}, r>0
$$

where $B\left(x_{0}, r\right)$ denotes the geodesic ball of radius $r$ and centered at $x_{0}, \operatorname{vol}\left(B\left(x_{0}, r\right)\right)$ denotes the volume of $B\left(x_{0}, r\right)$. Then $M^{n}$ is flat.

We briefly describe the proof of the theorem. Our method is via the study of the Yamabe flow on complete noncompact manifolds with nonnegative Ricci curvature. Suppose there exists such a manifold satisfying the quadratic decay condition (1.2). We evolve the metric by Yamabe flow. In section 2, we establish a short time existence result which concludes the solution either exists for all time or extracts to point in a finite time. Then in section 3 , by using the decay assumption we can derive a dedicate zero order estimate for the evolving metric. Particularly, this will imply that the solution of the Yamabe flow exists for all time. Finally in section 4, by combining the zero order estimate with Chow's Li-Yau-Hamilton inequality [C] on locally conformally flat manifolds, we are able to show that the initial metric is actually Ricci flat. However any locally conformally flat manifold with vanishing Ricci curvature must be flat.

\section{Short Time Existence.}

Let $M^{n}$ be an $n$-dimensional complete noncompact smooth Riemannian manifold with dimension $n \geq 3$. Suppose $M^{n}$ has bounded scalar curvature. Denote by $g_{i j}, R_{i j}$ and $R$ the metric tensor, the Ricci tensor and the scalar curvature. The Yamabe flow is the following evolution equation for 
the metric

$$
\begin{cases}\frac{\partial g_{i j}}{\partial t}=-R(x, t) g_{i j}(x, t), & x \in M^{n}, t>0 \\ g_{i j}(x, 0)=g_{i j}(x), & x \in M^{n}\end{cases}
$$

Write $g_{i j}(x, t)=[u(x, t)]^{\frac{4}{n-2}} g_{i j}(x)$ for some positive function $u(x, t)$. Then (2.1) can be written in the equivalent form

$$
\begin{cases}\frac{\partial u^{N}(x, t)}{\partial t}=(n-1) N\left[\Delta u(x, t)-\frac{n-2}{4(n-1)} R(x) u(x, t)\right], & \\ u(x, t)>0, & x \in M^{n}, t>0 \\ u(x, 0)=1, & x \in M^{n}, t>0 \\ & x \in M^{n},\end{cases}
$$

where $N=\frac{n+2}{n-2}, \triangle$ is the Laplace operator of the initial metric $g_{i j}(x)$, and $R(x)$ is the scalar curvature of the initial metric $g_{i j}(x)$.

To solve the problem (2.2), we first consider the corresponding Dirichlet problem

$$
\begin{cases}\frac{\partial u^{N}(x, t)}{\partial t}=(n-1) N\left[\Delta u(x, t)-\frac{n-2}{4(n-1)} R(x) u(x, t)\right], & \\ u(x, t)>0, & x \in \Omega, t>0 \\ u(x, t)=1, & x \in \Omega, t>0 \\ u(x, 0)=1, & x \in \partial \Omega, t>0 \\ & x \in \Omega,\end{cases}
$$

where $\Omega$ is a bounded smooth domain of $M^{n}$. In the following we will show that the solution of (2.3) exists for all times. Let us start with the local existence.

Lemma 2.1. For each $\delta \in(0,1)$ and $C>1$, there exists a constant $T>$ 0 , depending only on $\delta, C$ and the geometry of $\Omega$ such that if the initial data $u_{0}(x) \in C(\bar{\Omega}) \cap C^{2}(\Omega)$ satisfies

$$
\delta \leq u_{0}(x) \leq C, \quad x \in \Omega
$$

and

$$
\left.u_{0}\right|_{\partial \Omega}=1
$$


then the Dirichlet problem

$$
\begin{cases}\frac{\partial u^{N}(x, t)}{\partial t}=(n-1) N\left[\triangle u(x, t)-\frac{n-2}{4(n-1)} R\right. & (x) u(x, t)], \\ u(x, t)>0, & (x, t) \in Q \hat{=} \Omega \times(0, T), \\ u(x, t)=1, & (x, t) \in Q, \\ u(x, 0)=u_{0}(x), & (x, t) \in \partial \Omega \times(0, T), \\ & x \in \Omega,\end{cases}
$$

has a unique classical (i.e., $\left.C(\bar{\Omega}) \cap C^{2}(Q)\right)$ solution.

Proof. By applying the Leray-Schauder principle and the barrier argument in a standard way as in [LSU, $\S 6$, Chapter V], we only need to get a prior interior $C^{2, \alpha}$ estimate for the solution $u$. Assume that $u(x, t)$ achieves its maximum $u_{\max }(t)$ in the interior of $\Omega$. Then by a result of Hamilton (see Lemma 3.5 in [H1]) we have

$$
\frac{d u_{\max }^{N}(t)}{d t} \leq \frac{n+2}{4} \max _{x \in \Omega}|R(x)| \cdot u_{\max }(t)
$$

which implies that

$$
u_{\max }(t) \leq\left(C^{\frac{4}{n-2}}+\max _{x \in \Omega}|R(x)| \cdot t\right)^{\frac{n-2}{4}}, \quad \text { for } t>0 .
$$

Similarly the minimum $u_{\min }(t)$ of $u(\cdot, t)$ on $\Omega$ satisfies

$$
u_{\min }(t) \geq\left(\delta^{\frac{4}{n-2}}-\min _{x \in \Omega}|R(x)| \cdot t\right)^{\frac{n-2}{4}}, \quad \text { for } t>0 .
$$

Then there exists a positive constant $T$ depending only on $\delta, C$ and $\max _{x \in \Omega}|R(x)|$ such that

$$
\frac{1}{2} \delta \leq u(x, t) \leq 2 C, \quad \text { for } x \in \bar{\Omega}, t \in[0, T] .
$$

Thus we write the equation in the following form

$$
\frac{\partial u(x, t)}{\partial t}=\frac{n-1}{u^{N-1}(x, t)}\left[\triangle u(x, t)-\frac{n-2}{4(n-1)} R(x) u(x, t)\right],(x, t) \in Q .
$$

The uniform estimate (2.7) implies that the equation (2.8) is uniformly parabolic. So it follows from the Krylov-Safonov estimate [KS] that there is a prior interior $C^{\alpha}$ estimate for the solution $u(x, t)$ for some $0<\alpha<1$. And then we get a prior interior $C^{2, \alpha}$ estimate from Schauder theory. 
Proposition 2.2. The Dirichlet problem (2.3) has a unique classical solution for all time $t \in[0, \infty)$.

Proof. By lemma 2.1, we know that the problem (2.3) has a unique classical solution $u(x, t)$ on a maximal time interval $\left[0, t_{\max }\right)$ such that when $t_{\max }<$ $\infty$, then as $t \rightarrow t_{\max }$ either the minimum $u_{\min }(t)$ (of $u(x, t)$ on $\Omega$ ) tends to zero or the maximum $u_{\max }(t)$ (of $u(x, t)$ on $\Omega$ ) tends to infinity. Whenever the maximum $u_{\max }(t)$ is achieved in the interior of $\Omega$, it follows that

$$
\frac{d u_{\max }^{N}(t)}{d t} \leq \frac{n+2}{4} \max _{x \in \Omega}|R(x)| \cdot u_{\max }(t) .
$$

Thus

$$
u_{\max }(t) \leq\left[1+\max _{x \in \Omega}|R(x)| \cdot t\right]^{\frac{n-2}{4}}, \quad \forall \quad t \in\left[0, t_{\max }\right) .
$$

Suppose $t_{\max }<+\infty$, we then deduce that the minimum $u_{\min }(t)$ must tend to zero as $t \rightarrow t_{\max }$. Let us drop the restriction on positivity of the solution in (2.3). That is, we consider the problem

$$
\begin{cases}\frac{\partial u^{N}(x, t)}{\partial t}=(n-1) N\left[\Delta u(x, t)-\frac{n-2}{4(n-1)} R(x) u(x, t)\right], \\ u(x, t)=1, & x \in \Omega, t>0, \\ u(x, 0)=1, & x \in \Omega \Omega, t>0,\end{cases}
$$

By using DiBennedetto's estimate on the modulus of continuity of general porous medium equation [Di] (see also [Z]), we know from (2.9) that the solution $u(x, t)$ of $(\widetilde{2.3})$ can be extended continuously to $t_{\max }$. In the following we adapt an argument of $\mathrm{Ye}$ in $[\mathrm{Y}]$ to show that the extension is positive everywhere which will give a contradiction.

Since $u(x, t)=1$ on $\partial \Omega \times\left[0, t_{\max }\right]$, we can find a positive constant $r_{0}>$ 0 such that $u(x, t)$ is positive on the $r_{0}$-neighborhood of $\partial \Omega \times\left[0, t_{\max }\right]$. Without loss of generality, we may assume $r_{0}$ is less than the infimum of the injectivity radius of the points on $\Omega$. By the compactness of $\bar{\Omega}$, we only need to prove that $u\left(x, t_{\max }\right)$ is positive on the geodesic ball $B\left(x_{0}, \frac{r_{0}}{4}\right)$ whenever $u\left(x_{0}, t_{\max }\right)>0$. By continuity, there exist two small positive constants $\varepsilon, \delta$ such that

$$
u(x, t) \geq \varepsilon, \quad \text { for } x \in B\left(x_{0}, \delta\right), t \in\left[0, t_{\max }\right] .
$$


Let $\vec{e}$ be an arbitrary unit tangent vector at $x_{0}$. Denote by $P_{0}=\exp _{x_{0}}\left(\frac{r_{0}}{2} \vec{e}\right)$, $D=B\left(x_{0}, \delta\right) \cap \partial B\left(P_{0}, \frac{r_{0}}{2}\right)$, and $C$ to be the geodesic cone with tip $P_{0}$ and cap $D$. Let $r$ denote the distance to $P_{0}$ and set $S_{r}=\partial B\left(P_{0}, r\right)$ and $D_{r}=C \cap S_{r}$. Then the Laplacian operator of $g_{i j}(x)$ can be written

$$
\triangle=\frac{\partial^{2}}{\partial r^{2}}+H(r) \frac{\partial}{\partial r}+\triangle_{S_{r}}
$$

where $H(r)$ is the mean curvature function of $S_{r}=\partial B\left(P_{0}, r\right)$ and $\triangle_{S_{r}}$ is the Laplacian operator on $S_{r}$ for the induced metric. Let $\varphi(r, \cdot)$ denote the positive first eigenfunction of $r^{2} \triangle_{S_{r}}$ on $D_{r}$ with zero boundary values and the maximum one. Put $v=r^{b} \varphi$, where $b$ is to be determined. If we choose $b$ sufficiently large (depending only on $n, \varepsilon, \delta, \Omega, \max _{x \in \Omega}|R(X)|, t_{\max }$ ), then by the same calculation as in $[\mathrm{Y}]$,

$$
\triangle v-\frac{n-2}{4(n-1)} R v \geq 0, \quad \text { for } \quad r \leq \frac{r_{0}}{2} .
$$

Now choose a small $\varepsilon^{\prime}>0$ such that $\varepsilon^{\prime} v \leq u$ on $D \times\left[0, t_{\max }\right]$ and $C \times\{0\}$. The inequality $\varepsilon^{\prime} v \leq u$ also holds on the remaining part of the parabolic boundary of $C \times\left[0, t_{\max }\right]$, because $v$ vanishes there. Since we have

$$
\frac{\partial}{\partial t}\left[u^{N}-\left(\varepsilon^{\prime} v\right)^{N}\right] \geq(n-1) N\left[\triangle\left(u-\varepsilon^{\prime} v\right)-\frac{(n-2)}{4(n-1)} R\left(u-\varepsilon^{\prime} v\right)\right],
$$

on $C \times\left[0, t_{\max }\right]$, which implies $u \geq \varepsilon^{\prime} v$ on $C \times\left[0, t_{\max }\right]$ by the maximum principle. Consequently $u$ is positive along the geodesic $\exp _{x_{0}}(r \vec{e}), 0 \leq r \leq$ $\frac{r_{0}}{2}$. since $\vec{e}$ is arbitrary, we conclude that $u$ is positive in $B\left(x_{0}, \frac{r_{0}}{4}\right)$. This completes the proof of proposition.

The main result of this section is the following

Theorem 2.3. The Yamabe flow (2.1) has a smooth solution on a maximal time interval $\left[0, t_{\max }\right)$ with $t_{\max }>0$ such that either $t_{\max }=+\infty$, or the evolving metric contracts to a point at a finite time $t_{\max }$ in the sense that for any curve $\gamma$ on $M^{n}$, the length of $\gamma$ with respect to the evolving metric $g_{i j}(\cdot, t)$ tends to zero as $t \rightarrow t_{\max }$.

Proof. Let $\Omega_{1} \subset \Omega_{2} \subset \cdots$ be a sequence of exhausting bounded smooth domains of $M^{n}$. We solve the problem (2.3) for $\Omega=\Omega_{j}, j=1,2, \cdots$. to get a sequence of solutions $u_{j}(x, t)$ on $\Omega_{j} \times[0,+\infty), j=1,2, \cdots$. Let $x_{0}$ be 
any fixed point in $\Omega_{1}$. For simplicity, we may assume that the geodesic ball (w.r.t. the initial metric $\left.g_{i j}(x)\right) B\left(x_{0}, 1\right)$ is contained in $\Omega_{1}$.

Define

$$
\mu_{j}(t)=\inf _{(x, t) \in B\left(x_{0}, 1\right) \times[0, t]} u_{j}(x, t),
$$

and

$$
\mu(t)=\liminf _{j \rightarrow \infty} \mu_{j}(t) .
$$

By assumption, the initial metric $g_{i j}(x)$ has bounded scalar curvature . We know from (2.6) that $\mu(t)$ is positive for $t$ small enough. It is also clear that $\mu(t)$ is non-increasing in $t$. Thus we can distinguish two cases:

(i) $\mu(t)>0$ for all $t \in[0,+\infty)$;

(ii) there exists a finite time $t_{\max }>0$ such that $\mu(t)>0$ for $t \in\left[0, t_{\max }\right)$ and $\mu\left(t_{\max }\right)=0$.

First, we consider the case (i). For any fixed $T<+\infty$, we know from $(2.9)$ that $u_{j}(x, t)$ has a uniform upper bound on $M^{n} \times[0, T]$. Since $\mu(T)>0$, we still known from the proof of Proposition 2.2 that for any fixed compact subset $K$ of $M^{n}, u_{j}(x, t)$ has a positive lower bound on $K \times[0, T]$ for $j$ large enough. That says, the equation is uniformly parabolic on $K \times[0, T]$ Thus we can get the uniform $C^{\alpha}(0<\alpha<1)$ estimate from Krylov-Safonov estimate [KS] and then the uniform $C^{2, \alpha}(0<\alpha<1)$ estimate on $K \times[0, T]$. from Schauder theory for $j$. Hence by the arbitrariness of $K$ and $T$, it follows from a standard diagonal argument that (2.2) has a positive solution $u(x, t)$ on $M^{n} \times[0,+\infty)$.

Next, we consider the case (ii). That is, $0<t_{\max }<+\infty, \mu(t)>0$ on $\left[0, t_{\max }\right)$ and $\mu\left(t_{\max }\right)=0$. Denote by $t_{k}=\left(1-\frac{1}{k+1}\right) t_{\max }, k=1,2, \cdots$. Since $\mu\left(t_{1}\right)>0$, by repeating the above argument we can get a subsequence of $u_{j}$ so that it converges on every compact subset of $M^{n} \times\left[0, t_{1}\right)$. Similarly by $\mu\left(t_{2}\right)>0$, we can further get a subseqence from the previous subsequence so that it converges on every compact subset of $M^{n} \times\left[0, t_{2}\right)$. Then by repeating this procedure and the standard diagonal argument, we can get a smooth positive solution $u(x, t)$ defined on $M^{n} \times\left[0, t_{\max }\right)$, moreover by applying the result of DiBennedetto [Di] we can extend the solution $u(x, t)$ to $t_{\max }$ continuously. Again, the same argument in the proof of Proposition2.2 shows that $u\left(x, t_{\max }\right)$ must be identically zero on $M^{n}$. This implies that the length of any arc $\gamma$ with respect to the metric $g_{i j}(x, t)=(u(x, t))^{\frac{4}{n-2}} g_{i j}(x)$ tends to zero as $t \rightarrow t_{\max }$. 


\section{Zero Order Estimate.}

In this section we use the decay assumption on the curvature to drive a lower bound estimate for the solution of the Yamabe flow (2.2). Our argument is inspired by the work of Shi in [S].

Let $\left(M^{n}, g_{i j}\right)$ be a smooth complete noncompact Riemannian manifold with nonnegative Ricci curvature. Suppose the scalar curvature is bounded and satisfies (1.2).

From Theorem 2.3 we have a smooth solution $g_{i j}(x, t)$ of $(2.1)$ on a maximal time interval $\left[0, t_{\max }\right)$. Write the solution as $g_{i j}(x, t)=$ $(u(x, t))^{\frac{4}{n-2}} g_{i j}(x)$, where $u(x, t)$ is a positive solution of $(2.2)$ on $\left[0, t_{\max }\right)$.

We first present the following lemma.

Lemma 3.1. For any $1 \leq r<+\infty$, we have

$$
\begin{array}{r}
-\frac{1}{\operatorname{vol}\left(B\left(x_{0}, r\right)\right)} \int_{B\left(x_{0}, r\right)} \log u(x, t) d v \leq C\left[1-(\log u)_{\min }(t)\right] \cdot\left(\frac{t}{r^{2}}\right) \\
t>0
\end{array}
$$

where $(\log u)_{\min }(t)$ is the infimum of $\log u(x, t)$ for $x \in M^{n}$, and $C$ is some positive constant depending only on $n$.

Proof. Since the Ricci curvature of the initial metric $g_{i j}(x)$ is nonnegative, there exists a constant $C(n)>0$ depending only on the dimension such that for any fixed point $x_{0} \in M^{n}$ and any number $1 \leq r<+\infty$, there exists a smooth function $\varphi(x) \in C^{\infty}\left(M^{n}\right)$ such that

$$
\left\{\begin{array}{l}
\exp \left[-C(n)\left(1+\frac{d\left(x, x_{0}\right)}{r}\right)\right] \leq \varphi(x) \leq \exp \left[-\left(1+\frac{d\left(x, x_{0}\right)}{r}\right)\right] \\
|\nabla \varphi| \leq \frac{C(n)}{r} \varphi \\
|\triangle \varphi| \leq \frac{C(n)}{r^{2}} \varphi
\end{array}\right.
$$

where $d\left(x, x_{0}\right)$ is the distance between $x$ and $x_{0}$ in the metric $g_{i j}$. The construction of this kind of exponential decay function can be found in Shi $[\mathrm{S}]$ or Schoen-Yau [SY].

Recall the solution $u(x, t)$ is obtained by a sequence of approximating solutions $u_{j}(x, t), j=1,2, \cdots$, which solves the corresponding Dirichlet 
problem (2.3) for a sequence of exhausting domains $\Omega_{j}, j=1,2, \cdots$ By a direct computation,

$$
\begin{aligned}
\frac{\partial}{\partial t} \int_{\Omega_{j}} \varphi u_{j}^{N} d v= & \int_{\Omega_{j}} \varphi\left[(n-1) N \Delta u_{j}-\frac{(n+2)}{4} R u_{j}\right] d v \\
= & (n-1) N \int_{\Omega_{j}} \Delta \varphi \cdot u_{j} d v-\frac{n+2}{4} \int_{\Omega_{j}} R \varphi u_{j} d v \\
& +(n-1) N\left(\int_{\partial \Omega_{j}} \frac{\partial u_{j}}{\partial v} \varphi d s-\int_{\partial \Omega_{j}} u_{j} \frac{\partial \varphi}{\partial v} d s\right)
\end{aligned}
$$

where $v$ denotes the outer unit normal vector of $\partial \Omega_{j}$.

By noting that $R(x)$ is nonnegative, it follows from (2.3) and the maximal principle that $u_{j}(x, t) \leq 1$ on $\bar{\Omega}_{j} \times[0,+\infty)$. Since $\left.u_{j}\right|_{\partial \Omega_{j}}=1$, we deduce that $\frac{\partial u_{j}}{\partial \nu} \geq 0$ on $\partial \Omega_{j}$. To estimate the other boundary term, without loss of generality, we may assume $\Omega_{j}=B\left(x_{0}, j\right)$. Thus vol $\left(\partial \Omega_{j}\right) \leq C j^{n-1}$ for some constant $C$ (depending only on $n$ ) by the volume comparison theorem. And by (3.2) we have

$$
|\nabla \varphi| \leq \frac{C(n)}{r} e^{-\frac{j}{r}}, \quad \text { on } \quad \partial \Omega_{j}
$$

Then we get

$$
\left|\int_{\partial \Omega_{j}} u_{j} \frac{\partial \varphi}{\partial v} d s\right| \leq \frac{C \cdot C(n)}{r} j^{n-1} e^{-\frac{j}{r}} \rightarrow 0, \quad \text { as } j \rightarrow \infty .
$$

So by integrating (3.3) from 0 to $t$ and setting $j \rightarrow \infty$, we obtain

$$
\begin{aligned}
\int_{M^{n}} \varphi\left(1-u^{N}\right) d v & \leq \frac{C}{r^{2}} \int_{0}^{t} \int_{M^{n}} u \varphi d v+C \int_{0}^{t} \int_{M^{n}} u \varphi R d v \\
& \leq C t\left(\frac{1}{r^{2}} \int_{M^{n}} \varphi d v+\int_{M^{n}} \varphi R d v\right)
\end{aligned}
$$

for some positive constant $C$ depending only on $n$. 
From (1.2) and (3.2), we have ( see also $[\mathrm{S}]$ )

$$
\begin{aligned}
\int_{M^{n}} R \varphi d v \leq & \int_{M^{n}} R(x) e^{-\left(1+\frac{d\left(x, x_{0}\right)}{r}\right)} d v \\
= & \int_{B\left(x_{0}, r\right)} R(x) e^{-\left(1+\frac{d\left(x, x_{0}\right)}{r}\right)} d v \\
& +\sum_{k=0}^{\infty} \int_{B\left(x_{0}, 2^{k+1} r\right) \backslash B\left(x_{0}, 2^{k} r\right)} R(x) e^{-\left(1+\frac{d\left(x, x_{0}\right)}{r}\right)} d v \\
\leq & \frac{\varepsilon(r)}{r^{2}} \operatorname{vol}\left(B\left(x_{0}, r\right)\right)+\sum_{k=0}^{\infty} e^{-2^{k}} \frac{\varepsilon\left(2^{k+1} r\right)}{\left(2^{k+1} r\right)^{2}} \operatorname{vol}\left(B\left(x_{0}, 2^{k+1} r\right)\right) \\
\leq & \frac{C}{r^{2}}\left[\operatorname{vol}\left(B\left(x_{0}, r\right)\right)+\sum_{k=0}^{\infty} e^{\left.-2^{k} 2^{-2(k+1)} \operatorname{vol}\left(B\left(x_{0}, 2^{k+1} r\right)\right)\right] .}\right.
\end{aligned}
$$

By the volume comparison theorem,

$$
\begin{aligned}
& \sum_{k=0}^{\infty} e^{-2^{k}} 2^{-2(k+1)} \operatorname{vol}\left(B\left(x_{0}, 2^{k+1} r\right)\right) \\
& \leq \sum_{k=0}^{\infty} e^{-2^{k}} 2^{-2(k+1)} \cdot 2^{n(k+1)} \operatorname{vol}\left(B\left(x_{0}, r\right)\right) \\
& \leq C \operatorname{vol}\left(B\left(x_{0}, r\right)\right)
\end{aligned}
$$

thus (3.5) becomes

$$
\int_{M^{n}} R \varphi d v \leq \frac{C}{r^{2}} \operatorname{vol}\left(B\left(x_{0}, r\right)\right),
$$

for some positive constant $C$ depending only on $n$.

Similarly, by (3.2) we have

$$
C^{-1} \operatorname{vol}\left(B\left(x_{0}, r\right)\right) \leq \int_{M^{n}} \varphi d v \leq C \operatorname{vol}\left(B\left(x_{0}, r\right)\right)
$$

for some positive constant $C$ depending only on $n$.

Substituting (3.6) and (3.7) into (3.4) we deduce

$$
\int_{M^{n}} \varphi\left(1-u^{N}\right) d v \leq C \frac{t}{r^{2}} \operatorname{vol}\left(B\left(x_{0}, r\right)\right)
$$


for some positive constant $C$ depending only on $n$.

On the other hand, since $u \leq 1$ and $1-e^{x} \geq-\frac{x}{2}$ for $-1 \leq x \leq 0$, we get

$$
\begin{aligned}
\int_{M^{n}} \varphi\left(1-u^{N}\right) d v \geq & \int_{M^{n}} \varphi(1-u) d v \\
= & \int_{\{\log u \geq-1\}} \varphi\left(1-e^{\log u}\right) d v \\
& +\int_{\{\log u \leq-1\}} \varphi\left(1-e^{\log u}\right) d v \\
\geq & -\frac{1}{2} \int_{\{\log u \geq-1\}} \varphi \log u d v+\frac{1}{2} \int_{\{\log u \leq-1\}} \varphi d v .
\end{aligned}
$$

Note that the last two terms are positive. We deduce

$$
-\int_{\{\log u \geq-1\}} \varphi \log u d v \leq 2 \int_{M^{n}} \varphi\left(1-u^{N}\right) d v
$$

and

$$
\begin{aligned}
-\int_{\{\log u \leq-1\}} \varphi \log u d v & \leq-(\log u)_{\min }(t) \int_{\{\log u \leq-1\}} \varphi d v \\
& \leq-2(\log u)_{\min }(t) \int_{M^{n}} \varphi\left(1-u^{N}\right) d v .
\end{aligned}
$$

That is,

$$
-\int_{M^{n}} \varphi \log u d v \leq 2\left(1-(\log u)_{\min }(t)\right) \int_{M^{n}} \varphi\left(1-u^{N}\right) d v
$$

By (3.2), we have

$$
\begin{aligned}
-\int_{M^{n}} \varphi \log u d v & \geq-\int_{M^{n}} \log u \cdot e^{-C(n)\left(1+\frac{d\left(x_{0}, x\right)}{r}\right)} d v \\
& \geq-e^{-2 C(n)} \int_{B\left(x_{0}, r\right)} \log u d v .
\end{aligned}
$$

The combination of (3.8), (3.10) and (3.11) implies the estimate (3.1). 
We next want to estimate $-(\log u)_{\min }(t)$ in terms of $\frac{1}{\operatorname{vol}\left(B\left(x_{0}, r\right)\right)}$. $\int_{B\left(x_{0}, r\right)}(-\log u) d v$ by means of the positive Green function. Since in general the manifold $M^{n}$ is not necessary nonparabolic, we adapt a trick of Shi in [S] by considering a new manifold $\tilde{M}=M^{n} \times \mathbf{R}^{3}$, where $\mathbf{R}^{3}$ is equipped with the flat Euclidean metric and $\tilde{M}$ is equipped with the product metric. Let $x, y$ denote the points in $M^{n}$ and $\mathbf{R}^{3}$ respectively, $R(x)$ denote the scalar curvature of $M^{n}$ at $x$, and $\tilde{R}(x, y)$ denote the scalar curvature of $M^{n} \times \mathbf{R}^{3}$ at $(x, y)$. It is clear that $\tilde{R}(x, y)=R(x)$ for all $x \in M^{n}, y \in \mathbf{R}^{3}$. Thus the function $\tilde{u}$, defined by $\tilde{u}(x, y, t)=u(x, t)$ for $x \in M^{n}, y \in \mathbf{R}^{3}$ and $t>0$, is a solution of the following evolution equation.

$$
\begin{cases}\frac{\partial \tilde{u}^{N}(x, y, t)}{\partial t}=(n-1) N\left[\tilde{\triangle} \tilde{u}(x, y, t)-\frac{n-2}{4(n-1)} \tilde{R}(x, y) \tilde{u}(x, y, t)\right] \\ \tilde{u}(x, y, t)>0, & (x, y) \in \tilde{M}, t>0 \\ \tilde{u}(x, y, 0)=1, & (x, y) \in \tilde{M}, t>0 \\ & (x, y) \in \tilde{M}\end{cases}
$$

where $\tilde{\triangle}$ is the Laplacian operator of $\tilde{M}=M^{n} \times \mathbf{R}^{3}$.

We note that the manifold $\tilde{M}$ still has nonnegative Ricci curvature and

$$
B_{M^{n}}\left(x_{0}, \frac{r}{2}\right) \times B_{\mathbf{R}^{3}}\left(y_{0}, \frac{r}{2}\right) \subset \tilde{B}\left(\left(x_{0}, y_{0}\right), r\right) \subset B_{M^{n}}\left(x_{0}, r\right) \times B_{\mathbf{R}^{3}}\left(y_{0}, r\right) .
$$

Then by the volume comparison theorem, we have

$$
C(n)\left(\frac{r_{2}}{r_{1}}\right)^{3} \leq \frac{\operatorname{vol} \tilde{B}\left(\left(x_{0}, y_{0}\right), r_{2}\right)}{\operatorname{vol} \tilde{B}\left(\left(x_{0}, y_{0}\right), r_{1}\right)} \leq\left(\frac{r_{2}}{r_{1}}\right)^{n+3}, \text { for } r_{1} \leq r_{2}
$$

and

$$
\begin{aligned}
\int_{\tilde{B}\left(\left(x_{0}, y_{0}\right), r\right)} \tilde{R}(x, y) d \tilde{v} & \leq \int_{B_{M^{n}}\left(x_{0}, r\right) \times B_{\mathbf{R}^{3}}\left(y_{0}, r\right)} \tilde{R}(x, y) d \tilde{v} \\
& \leq C r^{3} \int_{B_{M^{n}\left(x_{0}, r\right)}} R(x) d v \\
& \leq \tilde{C} \frac{\varepsilon(r)}{r^{2}} \operatorname{vol} \tilde{B}\left(\left(x_{0}, y_{0}\right), r\right)
\end{aligned}
$$

that is,

$$
\frac{r^{2}}{\operatorname{vol} \tilde{B}\left(\left(x_{0}, y_{0}\right), r\right)} \int_{\tilde{B}\left(\left(x_{0}, y_{0}\right), r\right)} \tilde{R}(x, y) d \tilde{v} \leq \tilde{C} \varepsilon(r)
$$


where $C, C(n)$ and $\tilde{C}$ are positive constants depending only on $n$. It is also clear that to estimate $-(\log u)_{\min }(t)$ in terms of $\frac{1}{\operatorname{vol}\left(B\left(x_{0}, r\right)\right.} \cdot \int_{B\left(x_{0}, r\right)}(-\log u) d v$ is equivalent to estimate $-(\log \tilde{u})_{\min }(t)$ in terms of $\frac{1}{\operatorname{vol} \tilde{B}\left(\left(x_{0}, y_{0}\right), r\right)}$ $\tilde{B}\left(\left(x_{0}, y_{0}\right), r\right)$

$\tilde{u}(x, y, t)$ of (3.12) with the manifold $\tilde{M}$ satisfying (3.13) and (3.14). For simplicity, we drop the symbol " $\sim$ " and the variable " $y$ " off.

Lemma 3.2. There exist a positive constant $C(n)$ and a positive function $\widetilde{\varepsilon}(r)$ defined on $[1,+\infty)$ satisfying $\widetilde{\varepsilon}(r) \rightarrow 0$ as $r \rightarrow+\infty$ such that

$$
\begin{aligned}
& \log u\left(x_{0}, t\right) \\
& \geq C\left[-\widetilde{\varepsilon}(r) \log r-\sup _{x \in M^{n}} R(x)+\frac{1}{\operatorname{vol}\left(B\left(x_{0}, r\right)\right)} \int_{B\left(x_{0}, r\right)} \log u(x, t) d v\right],
\end{aligned}
$$

for all $t>0$ and $r \geq 1$.

Proof. Since the Ricci curvature of $M$ is nonnegative and (3.13) holds, we know from [LY] ( see also Lemma 6.6 of $[\mathrm{S}]$ ) that there exists a positive Green function $G\left(x_{0}, x\right)$ satisfying

$$
\frac{d^{2}\left(x_{0}, x\right)}{C \operatorname{vol}\left(B\left(x_{0}, d\left(x_{0}, x\right)\right)\right.} \leq G\left(x_{0}, x\right) \leq \frac{C d^{2}\left(x_{0}, x\right)}{\operatorname{vol}\left(B\left(x_{0}, d\left(x_{0}, x\right)\right)\right.}, \quad x \in M
$$

and

$$
\left|\nabla G\left(x_{0}, x\right)\right| \leq \frac{C d\left(x_{0}, x\right)}{\operatorname{vol}\left(B\left(x_{0}, d\left(x_{0}, x\right)\right)\right.}, \quad x \in M .
$$

Here and in the following we denote $C$ to be variant positive constant depending only on $n$. For any $\alpha>0$, we define

$$
\Omega_{\alpha}=\left\{x \in M \mid G\left(x_{0}, x\right)>\alpha\right\} .
$$

It is easy to see from (3.13) and (3.16) that

$$
\frac{1}{C} \cdot \frac{1}{d\left(x_{0}, x\right)^{n+1} \operatorname{vol}\left(B\left(x_{0}, 1\right)\right)} \leq G\left(x_{0}, x\right) \leq \frac{C}{d\left(x_{0}, x\right) \operatorname{vol}\left(B\left(x_{0}, 1\right)\right)}
$$


for $d\left(x_{0}, x\right) \geq 1$. Thus $\bar{\Omega}_{\alpha}$ is compact subset of $M$ and $\partial \Omega_{\alpha}=\{x \in$ $\left.M \mid G\left(x_{0}, x\right)=\alpha\right\}$. Moreover for each $\alpha>0$ there is a $d(\alpha)$ such that

$$
\frac{d^{2}(\alpha)}{\operatorname{vol}\left(B\left(x_{0}, d(\alpha)\right)\right.}=\alpha \text { and } d(\alpha) \rightarrow 0 \text { as } \alpha \rightarrow \infty .
$$

This implies that for any $x \in \partial \Omega_{\alpha}$;

$$
\frac{d^{2}\left(x_{0}, x\right)}{C \operatorname{vol}\left(B\left(x_{0}, d\left(x_{0}, x\right)\right)\right.} \leq \frac{d^{2}(\alpha)}{\operatorname{vol}\left(B\left(x_{0}, d(\alpha)\right)\right.} \leq \frac{C d^{2}\left(x_{0}, x\right)}{\operatorname{vol}\left(B\left(x_{0}, d\left(x_{0}, x\right)\right)\right.},
$$

and then

$$
\frac{\operatorname{vol}\left(B\left(x_{0}, d(\alpha)\right)\right.}{C \operatorname{vol}\left(B\left(x_{0}, d\left(x_{0}, x\right)\right)\right)} \leq \frac{d^{2}(\alpha)}{d^{2}\left(x_{0}, x\right)} \leq \frac{C \operatorname{vol}\left(B\left(x_{0}, d(\alpha)\right)\right)}{\operatorname{vol}\left(B\left(x_{0}, d\left(x_{0}, x\right)\right)\right)} .
$$

Combining (3.13) and (3.19) we get

$$
C^{-1} d(\alpha) \leq d\left(x_{0}, x\right) \leq C d(\alpha), \quad \text { for } x \in \partial \Omega_{\alpha},
$$

and

$$
B\left(x_{0}, C^{-1} d(\alpha)\right) \subset \Omega_{\alpha} \subset B\left(x_{0}, C d(\alpha)\right) .
$$

Note by the Green formula, it follows

$$
\begin{aligned}
\log u\left(x_{0}, t\right)= & \int_{\Omega_{\alpha}}\left(\alpha-G\left(x_{0}, x\right)\right) \triangle \log u(x, t) d v \\
& -\int_{\partial \Omega_{\alpha}} \log u(x, t) \cdot \frac{\partial G\left(x_{0}, x\right)}{\partial \nu} d s,
\end{aligned}
$$

where $\nu$ denotes the outer unit normal vector of $\partial \Omega_{\alpha}$.

By a direct computation (see also Lemma 2.2 in [C]) we known that the scalar curvature $R(x, t)$ of the solution evolves by

$$
\frac{\partial R(x, t)}{\partial t}=(n-1) \triangle_{t} R(x, t)+(R(x, t))^{2},
$$

where $\triangle_{t}$ is the Laplacian operator with respect to the metric $g_{i j}(x, t)$. Since the scalar curvature of the initial metric is nonnegative, we know from the maximum principle that $R(x, t) \geq 0$, for $x \in M$ and $t>0$. Thus by the fact that $g_{i j}(x, t)=(u(x, t))^{\frac{4}{n-2}} g_{i j}(x)$ for $x \in M$ and $t>0$, we obtain

$$
\triangle u(x, t)-\frac{(n-2)}{4(n-1)} R(x) u(x, t) \leq 0, \quad \text { for } x \in M, t>0 .
$$


This concludes that

$$
\triangle \log u(x, t) \leq \frac{n-2}{4(n-1)} R(x), \quad \text { for } \quad x \in M, t>0
$$

Substituting (3.23) into (3.22), we get

$$
\begin{aligned}
\log u\left(x_{0}, t\right) \geq & \frac{n-2}{4(n-1)} \int_{\Omega_{\alpha}}\left(\alpha-G\left(x_{0}, x\right)\right) R(x) d v \\
& -\int_{\partial \Omega_{\alpha}} \log u(x, t) \cdot \frac{\partial G\left(x_{0}, x\right)}{\partial \nu} d s \\
\geq & -\frac{n-2}{4(n-1)} \int_{\Omega_{\alpha}} G\left(x_{0}, x\right) R(x) d v \\
& +\int_{\partial \Omega_{\alpha}} \log u(x, t) \cdot\left|\nabla G\left(x_{0}, x\right)\right| d s .
\end{aligned}
$$

We estimate the last two terms as follows:

For any $\alpha>0$, by (3.16), (3.20) and (3.21), we obtain

$$
\begin{aligned}
\int_{\Omega_{\alpha}} G\left(x_{0}, x\right) R(x) d v \leq & \int_{B\left(x_{0}, C d(\alpha)\right)} G\left(x_{0}, x\right) R(x) d v \\
\leq & \int_{B\left(x_{0}, 1\right)} G\left(x_{0}, x\right) R(x) d v \\
& +\sum_{k=1}^{s} \int_{B\left(x_{0}, 2^{k}\right) \backslash B\left(x_{0}, 2^{k-1}\right)} G\left(x_{0}, x\right) R(x) d v,
\end{aligned}
$$


A Gap Theorem ...

where $s$ is the integer satisfying $2^{s-1} \leq C d(\alpha) \leq 2^{s}$, and

$$
\begin{aligned}
& \int_{B\left(x_{0}, 1\right)} G\left(x_{0}, x\right) R(x) d v \\
& \leq\left(\sup _{x \in M} R(x)\right) \int_{B\left(x_{0}, 1\right)} G\left(x_{0}, x\right) d v \\
& \leq\left(\sup _{x \in M} R(x)\right) \sum_{k=0}^{\infty} \int_{B\left(x_{0}, 2^{-k}\right) \backslash B\left(x_{0}, 2^{-k-1}\right)} \frac{C d^{2}\left(x_{0}, x\right)}{\operatorname{vol}\left(B\left(x_{0}, d\left(x_{0}, x\right)\right)\right)} d v \\
& \leq C\left(\sup _{x \in M} R(x)\right) \sum_{k=0}^{\infty} \frac{2^{-2 k}}{\operatorname{vol}\left(B\left(x_{0}, 2^{-k-1}\right)\right)} \operatorname{vol}\left(B\left(x_{0}, 2^{-k}\right)\right) \\
& \leq C\left(\sup _{x \in M} R(x)\right) \\
& \sum_{k=1}^{s} \int_{B\left(x_{0}, 2^{k}\right) \backslash B\left(x_{0}, 2^{k-1}\right)} G\left(x_{0}, x\right) R(x) d v \\
& \leq \sum_{k=1}^{s} \int_{B\left(x_{0}, 2^{k}\right) \backslash B\left(x_{0}, 2^{k-1}\right)} \frac{C d^{2}\left(x_{0}, x\right)}{\operatorname{vol}\left(B\left(x_{0}, d\left(x_{0}, x\right)\right)\right)} R(x) d v \\
& \leq C \sum_{k=1}^{s} \frac{2^{2 k}}{\operatorname{vol}\left(B\left(x_{0}, 2^{k-1}\right)\right)} \int_{B\left(x_{0}, 2^{k}\right)} R(x) d v \\
& \leq C \sum_{k=1}^{s} \frac{2^{2 k}}{\operatorname{vol}\left(B\left(x_{0}, 2^{k-1}\right)\right)} \cdot \frac{\varepsilon\left(2^{k}\right)}{2^{2 k}} \operatorname{vol}\left(B\left(x_{0}, 2^{k}\right)\right) \\
& \leq C \sum_{k=1}^{s} \varepsilon\left(2^{k}\right) \\
& \leq C\left(\frac{1}{s} \sum_{k=1}^{s} \varepsilon\left(2^{k}\right)\right) \cdot(1+\log d(\alpha)),
\end{aligned}
$$

thus

$$
\int_{\Omega_{\alpha}} G\left(x_{0}, x\right) R(x) d v \leq C\left[\sup _{x \in M} R(x)+\frac{1}{s} \sum_{k=1}^{s} \varepsilon\left(2^{k}\right) \cdot(1+\log d(\alpha))\right] .
$$

By (3.17), (3.13) and (3.20), we have

$$
\int_{\partial \Omega_{\alpha}} \log u(x, t) \cdot\left|\nabla G\left(x_{0}, x\right)\right| d s \geq \frac{C d(\alpha)}{\operatorname{vol}\left(B\left(x_{0}, d(\alpha)\right)\right.} \int_{\partial \Omega_{\alpha}} \log u(x, t) d s .
$$


We know from the definition of $d(\alpha)$ that for $\beta \geq \alpha$,

$$
\frac{\beta}{\alpha}=\left(\frac{d^{2}(\beta)}{d^{2}(\alpha)}\right) /\left(\frac{\operatorname{vol}\left(B\left(x_{0}, d(\beta)\right)\right.}{\operatorname{vol}\left(B\left(x_{0}, d(\alpha)\right)\right.}\right),
$$

and by (3.13)

$$
\left(\frac{d(\alpha)}{d(\beta)}\right)^{n+1} \leq \frac{\beta}{\alpha} \leq C \cdot \frac{d(\alpha)}{d(\beta)} .
$$

Thus by integrating (3.26) from $\alpha$ to $2 \alpha$, we get

$$
\begin{aligned}
& \int_{\alpha}^{2 \alpha} \int_{\partial \Omega_{\beta}} \log u(x, t) \cdot\left|\nabla G\left(x_{0}, x\right)\right| d s d \beta \\
& \geq \frac{C d(\alpha)}{\operatorname{vol}\left(B\left(x_{0}, d(\alpha)\right)\right.} \int_{\alpha}^{2 \alpha} \int_{\partial \Omega_{\beta}} \log u(x, t) d s d \beta .
\end{aligned}
$$

Since $d \beta=\frac{\partial G}{\partial \nu} d \nu$, we have

$$
d s d \beta=\left|\frac{\partial G}{\partial \nu}\right| d s|d \nu| \leq|\nabla G| d v \leq C \frac{d(\beta)}{\operatorname{vol}\left(B\left(x_{0}, d(\beta)\right)\right.} d v, \quad \text { on } \partial \Omega_{\beta} .
$$

Then (3.28) becomes

$$
\begin{aligned}
& \int_{\alpha}^{2 \alpha} \int_{\partial \Omega_{\alpha}} \log u(x, t) \cdot\left|\nabla G\left(x_{0}, x\right)\right| d s d \beta \\
& \geq C\left(\frac{d(\alpha)}{\operatorname{vol}\left(B\left(x_{0}, d(\alpha)\right)\right.}\right)^{2} \int_{\Omega_{\alpha} \backslash \Omega_{2 \alpha}} \log u(x, t) d v \\
& \geq C \frac{\alpha}{\operatorname{vol}\left(B\left(x_{0}, C d(\alpha)\right)\right.} \int_{B\left(x_{0}, C d(\alpha)\right.} \log u(x, t) d v .
\end{aligned}
$$

By integrating (3.24) from $\alpha$ to $2 \alpha$ and substituting the estimates (3.25) and (3.29) into the expression, we obtain

$$
\begin{aligned}
\log u\left(x_{0}, t\right) \geq & C \frac{1}{\operatorname{vol}\left(B\left(x_{0}, C d(\alpha)\right)\right)} \int_{B\left(x_{0}, C d(\alpha)\right)} \log u(x, t) d v \\
& -C\left[\sup _{x \in M} R(x)+\frac{1}{s} \sum_{k=1}^{s} \varepsilon\left(2^{k}\right) \cdot(1+\log d(\alpha))\right] .
\end{aligned}
$$


Finally for any $r>0$, set $\alpha=\frac{r^{2}}{\operatorname{vol}\left(B\left(x_{0}, r\right)\right)}$. From the definition of $d(\alpha)$, we have

$$
\frac{r^{2}}{\operatorname{vol}\left(B\left(x_{0}, r\right)\right)}=\frac{d^{2}(\alpha)}{\operatorname{vol}\left(B\left(x_{0}, d(\alpha)\right)\right)}
$$

which together with (3.13) implies

$$
C^{-1} r \leq d(\alpha) \leq C r .
$$

It is easy to know that

$$
\frac{1}{s} \sum_{k=1}^{s} \varepsilon\left(2^{k}\right) \rightarrow 0 \quad \text { as } \quad d(\alpha) \rightarrow+\infty,
$$

because $\varepsilon(r) \rightarrow 0$ as $r \rightarrow+\infty$. Therefore by combining (3.30), (3.31) and (3.32) we get the desired estimate (3.15) with $\tilde{\varepsilon}(r)$ defined by $\frac{1}{s} \sum_{k=1}^{s} \varepsilon\left(2^{k}\right)$.

The combination of Lemma 3.1 and Lemma 3.2 is the following lower bound estimate for the solution of (2.2).

Theorem 3.3. Suppose $\left(M^{n}, g_{i j}\right)$ is a smooth $n$-dimensional $(n \geq 3)$ complete, noncompact Riemannian manifold with nonnegative Ricci curvature. Suppose also the scalar curvature is bounded and satisfies (1.2). Then the solution $u(x, t)$ of $(2.2)$ obtained in section 2 exists for all times and satisfies

$$
(\log u)_{\min }(t) \geq-\widetilde{\varepsilon}(r) \log r-C\left[1+\sup _{x \in M} R(x)+\frac{t}{r^{2}}(\log u)_{\min }(t)\right]
$$

for all $t>0$ and $1 \leq r<+\infty$, where $C$ is some positive constant depending only on $n$ and $\widetilde{\varepsilon}(r)$ is some positive function defined on $[1,+\infty)$ satisfying $\widetilde{\varepsilon}(r) \rightarrow 0$ as $r \rightarrow+\infty$.

\section{The Proof of the Main Theorem.}

In this section we will give a proof for the main theorem based on the lower bound estimate of the previous section and the Li-Yau-Hamilton inequality of Chow $[\mathrm{C}]$ on locally conformally flat manifolds. We first recall the LiYau-Hamilton inequality as follows.

Theorem 4.1 (Chow). Suppose $\left(M^{n}, g_{i j}\right)$ is an $n$-dimensional $(n \geq 3)$ complete locally conformally flat Riemannian manifold with bounded and 
nonnegative Ricci curvature. Let $R(x, t)$ be the scalar curvature of the solution of the Yamabe flow with $g_{i j}$ as initial metric. Then we have

$$
\frac{\partial R}{\partial t}+\langle\nabla R, X\rangle_{t}+\frac{1}{2(n-1)} R_{i j} X^{i} X^{j}+\frac{R}{t} \geq 0
$$

for any vector $X$. Particularly if let $X=-\frac{\nabla R}{R}$ in (4.1), we have

$$
\frac{\partial R}{\partial t}+\frac{R}{t} \geq \frac{1}{2} \frac{|\nabla R|_{t}^{2}}{R}
$$

where $\langle\cdot, \cdot\rangle_{t}$ and $|\cdot|_{t}$ are the inner product and norm with respect to the evolving metric $g_{i j}(x, t)$.

In his paper $[\mathrm{C}]$, Chow proved the above theorem for compact locally comformally flat manifolds with positive Ricci curvature. However, by a perturbation argument as in [H2], it is pretty clear that the Li-Yau-Hamilton inequality actually holds for complete locally conformally flat manifolds with nonnegative Ricci curvature.

Proof of the Main Theorem.

Suppose $\left(M^{n}, g_{i j}\right)$ is an $n$-dimensional $(n \geq 3)$ complete noncompact locally conformally flat manifold with nonnegative Ricci curvature. Suppose also the scalar curvature is bounded and satisfies (1.2). We evolve the metric by the Yamabe flow (2.1). From Theorem 3.3 we know that the solution exists for all time $t \geq 0$ and satisfies the lower bound estimate (3.33). By setting $t=r$ in (3.33), it follows

$$
\lim _{t \rightarrow+\infty} \frac{-(\log u)_{\min }(t)}{\log t}=0 .
$$

Thus by the evolution equation $(2.1)$ and $g_{i j}(x, t)=(u(x, t))^{\frac{4}{n-2}} g_{i j}(x)$, we have

$$
\int_{0}^{t} R(x, \tau) d \tau=-\frac{4}{n-2} \log u(x, t) \leq-\frac{4}{n-2}(\log u)_{\min }(t) .
$$

Let $t>1$. It follows from (4.2) that

$$
R(x, \tau) \geq R(x, \sqrt{t}) \frac{\sqrt{t}}{\tau}, \quad \text { for } \tau \geq \sqrt{t} .
$$


Then we obtain

$$
\begin{aligned}
\int_{0}^{t} R(x, \tau) d \tau & \geq \int_{\sqrt{t}}^{t} R(x, \tau) d \tau \\
& \geq R(x, \sqrt{t}) \sqrt{t} \int_{\sqrt{t}}^{t} \frac{d \tau}{\tau} \\
& \geq \frac{1}{2} R(x, \sqrt{t}) \sqrt{t} \log t
\end{aligned}
$$

By combining (4.4) and (4.5), we have

$$
R(x, \sqrt{t}) \sqrt{t} \leq-\frac{8}{n-2} \frac{(\log u)_{\min }(t)}{\log t}
$$

which together with (4.3) implies

$$
\lim _{t \rightarrow+\infty} R(x, \sqrt{t}) \sqrt{t}=0 .
$$

But (4.2) tells us that $R(x, \sqrt{t}) \sqrt{t}$ is nondecreasing in time. This shows that $R(x, t) \equiv 0$ for all $x \in M, t>0$. Therefore the manifold $M$ with the initial metric must be flat.

\section{References.}

[BKN] S. Bando, A. Kasue, and H. Nakajima, On a construction of coordinates at infinity on manifolds with fast curvature decay and maximal volume growth, Invent. Math., 97 (1989), 313-349.

[C] B. Chow, The Yamabe flow on locally conformally flat manifolds with positive Ricci curvature, Comm. Pure Appl. Math., XIV (1992), 1003-1014.

[Di] E. Di Bennedetto, Continuity of weak solutions to a general porous medium equation, Indiana Univ. Math. J., 32 (1983), 83-118.

[Dr] G. Drees, Asymptotically flat manifold of nonnegative curvature, Differential Geom. Appl., 4 (1994), 77-90. 
[ESS] J. Eschenburg, V. Schroeder and M. Strake, Curvature at infinity of open nonnegatively curved manifold, J. Differential Geometry, 30 (1989), 155-166.

[GW] R.E. Greene and H. Wu, Gap theorems for noncompact Riemannian manifolds, Duke Math. J., 49 (1982), 731-756.

[H1] R.S. Hamilton, Four-manifolds with positive curvature operator, J. Differential Geometry, 24 (1986), 153-179.

[H2] R.S. Hamilton, The Harnack estimate for the Ricci flow, J. Differential Geom., 37 (1993), 225-243.

[KS] N.V. Krylov and M.V. Safonov, A certain property of solutions of parabolic equations with measurable coefficients, Math. USSR-Izv., 16 (1981), 151-164.

[LSU] O.A. Ladyzhenskaya, V.A. Solonnikov and N.N. Uralćeva, Linear and quasilinear equations of parabolic type, Amer. Math. Society, Providence, RI, 1968.

[LY] P. Li and S.T. Yau, On the parabolic kernel of the Schrödinger operator, Acta Math, 156 (1986), 153-201.

[MSY] N. Mok, Y.T. Siu and S.T. Yau, The Poincare-Lelong equation on complete Kähler manifolds, Compositio Math., 44 (1981), 183-218.

[N] L. Ni, Vanishing theorems on complete Kähler manifolds and their applications, J. Differential Geom. 50(1998) 89-122.

[P] P. Petersen, Convergence theorems in Riemannian geometry, in Comparison Geometry, MSRI Publications, 30, 1997.

[SY] R. Schoen and S.T. Yau, Lectures on differential geometry, in 'Conference proceedings and Lecture Notes in Geometry and Topology,' 1, International Press Publications, 1994.

[S] W.X. Shi, Ricci flow and the uniformization on complete noncompact Kähler manifolds, J. Differential Geometry, 45 (1997), 94-220.

[TY] G. Tian and S.T. Yau, Complete Kähler manifolds with zero Ricci curvature, 1, J. Amer. Math. Soc., 3 (1990), 579-610.

[Y] R. Ye, Global existence and convergence of Yamabe flow, J. Differential Geom. 39(1994), 35-50. 
[Z] W.P. Ziemer, Interior and boundary continuity of weak solutions of degenerate parabolic equations, Trans. Amer. Math. Soc., 271 (1982), 733-748.

Department of Mathematics

ZHONGSHAN UNIVERSITY

Guangzhou 510275, P. R. China

E-mail address: stszxp@zsu.edu.cn

ReCeived January 24, 2000. 